\title{
AN ITERATIVE METHOD FOR THE RECONSTRUCTION OF TWO-DIMENSIONAL DENSITY DISTRIBUTIONS
}

\author{
M. BREMER \\ Astronomisches Institut der Universität, Waldhäuserstraße 64, D-7400 Tübingen \\ M. GREWING \\ Astronomisches Institut der Universität, Waldhäuserstraße 64, D-7400 Tübingen \\ and
}

Institute for Radioastronomy at Millimeter Wavelengths (IRAM), 300, rue de la Piscine, Domaine Universitaire, F-38406 St .Martin d'Heres, France

\begin{abstract}
As hydrodynamic models of planetary nebulae advance from 1D to $2 \mathrm{D}$ calculations, it becomes desirable to make the same step for reconstruction techniques, which aim at deriving from the $2 \mathrm{D}$ intensity distributions of $\mathrm{PNe}$ images their 3D structure. A basic step is the determination of a symmetry axis and its orientation in space which can be described by two angles, one measured in the plane of the sky, and one measured with respect to the tangential plane. While the first one can be determined from the images, e.g. by applying the criterion of maximum normalized correlation between the object halves, which we found to yield the best results, the second angle is treated as a free parameter (see below). The steps of the iterative reconstruction algorithm are the following (with image $(x, y)=$ input image):
\end{abstract}

1. Fill a 3D kartesian density grid(xyz) (random or continuous), observing the constraint

$\int \operatorname{grid}(x y z) d z=\operatorname{image}(x, y)$, where $\mathrm{z}$ is along the line of sight.

2. Transform to cylindrical symmetry system $\left(\mathrm{r}, \mathrm{z}^{\prime}, \varphi\right)$ and read out density $\left(\mathrm{r}, \mathrm{z}^{\prime}\right)$ averaging over $\varphi$.

3. Return density(r,z') into $\operatorname{grid}(\mathrm{xyz})$ and normalize the $\int \operatorname{grid}(x y z) d z$ to match the input image.

4. Return to (2.) as long as two subsequent density(r,z') estimations differ by more than a specified limit.

This algorithm can be applied to CCD images without previous smoothing and cannot produce negative densities like the inverted Abel Transform (IAT). However, the results are not unique. A special status for the observer's point of view (condensations followed by low density regions are allowed in the line of sight) can be included or suppressed by the careful choice of weighting functions $g(\varphi)$ at $\varphi$ averaging (for example, $g(\varphi)=|\sin \varphi|^{m}: m=0$ special, increasing $m$ less special). An entirely different, noniterative approach using the IAT and deconvolving the tilt was constructed and tested with less success. This algorithm turned out to be extremely instable (oscillations, negative densities) and was therefore given up. Using the iterative algorithm with monochromatic CCD $\mathrm{H} \alpha$ images as input, we present density distributions for several objects with different morphologies under different assumed tilt angles. Unfortunately, several of them are 'plausible' under hydrodynamical considerations, i.e. the uniqueness problem remains to be solved. 